\title{
Behavioral Economics Interventions to Improve Outpatient Antibiotic Prescribing for Acute Respiratory Infections: a Cost-Effectiveness Analysis
}

\author{
Cynthia L. Gong, Pharm.D. ', Kenneth M. Zangwill, M.D. ${ }^{2}$, Joel W. Hay, Ph.D. ', \\ Daniella Meeker, Ph.D. ${ }^{1,3}$, and Jason N. Doctor, Ph.D. ${ }^{1}$
}

'University of Southern California Leonard D. Schaeffer Center for Health Policy \& Economics, Los Angeles, CA, USA; ${ }^{2}$ Los Angeles Biomedical Research Institute at Harbor-UCLA Medical Center, Los Angeles, CA, USA; ${ }^{3}$ University of Southern California Keck School of Medicine, Los Angeles, CA, USA.

BACKGROUND: Behavioral economics interventions have been shown to effectively reduce the rates of inappropriate antibiotic prescriptions for acute respiratory infections (ARIs).

OBJECTIVE: To determine the cost-effectiveness of three behavioral economic interventions designed to reduce inappropriate antibiotic prescriptions for ARIs.

DESIGN: Thirty-year Markov model from the US societal perspective with inputs derived from the literature and CDC surveillance data.

SUBJECTS: Forty-five-year-old adults with signs and symptoms of ARI presenting to a healthcare provider.

INTERVENTIONS: (1) Provider education on guidelines for the appropriate treatment of ARIs; (2) Suggested Alternatives, which utilizes computerized clinical decision support to suggest non-antibiotic treatment choices in lieu of antibiotics; (3) Accountable Justification, which mandates free-text justification into the patient's electronic health record when antibiotics are prescribed; and (4) Peer Comparison, which sends a periodic email to prescribers about his/her rate of inappropriate antibiotic prescribing relative to clinician colleagues.

MAIN MEASURES: Discounted costs, quality-adjusted life years (QALYs), and incremental cost-effectiveness ratios.

KEY RESULTS: Each intervention has lower costs but higher QALYs compared to provider education. Total costs for each intervention were \$178.21, \$173.22, $\$ 172.82$, and $\$ 172.52$, and total QALYs were 14.68 , $14.73,14.74$, and 14.74 for the control, Suggested Alternatives, Accountable Justification, and Peer Comparison groups, respectively. Results were most sensitive to the quality-of-life of the uninfected state, and the likelihood and costs for antibiotic-associated adverse events.

CONCLUSIONS: Behavioral economics interventions can be cost-effective strategies for reducing inappropriate antibiotic prescriptions by reducing healthcare resource utilization.

KEY WORDS: cost-effectiveness; healthcare administration; physician behavior; infectious disease.

Received July 14, 2017

Revised March 6, 2018

Accepted April 13, 2018

Published online May 8, 2018
J Gen Intern Med 34(6):846-54

DOI: $10.1007 / \mathrm{s} 11606-018-4467-\mathrm{x}$

(c) Society of General Internal Medicine 2018

\section{INTRODUCTION}

In the USA, it is estimated that more than $50 \%$ of outpatientprescribed antibiotics are inappropriate, predominantly among patients seeking treatment for acute respiratory infections (ARIs) caused by viruses. Such unnecessary antibiotic use leads to increased risk of adverse events and emergency department (ED) visits for such events and additional financial costs to the healthcare system. ${ }^{1-3}$ Furthermore, excess antibiotic use contributes to the ever-increasing problem of antibiotic resistance. ${ }^{4-6}$ The Centers for Disease Control and Prevention (CDC) notes that the single most important action needed to slow the spread of antibiotic-resistant infections is to reduce the amount of inappropriate and unnecessary antibiotic use in humans and animals. ${ }^{7}$ A large body of work describes various attempts to curb inappropriate antibiotic prescribing through traditional interventions such as physician and patient education, electronic clinical decision support, and financial incentives. These have only resulted in modest reductions in antibiotic prescribing rates for nonbacterial ARIs. ${ }^{8,9}$

An alternative approach to changing prescribing behavior applies ideas from the behavioral sciences, using social cues and subtle changes in the clinic environment to influence clinical decision making. ${ }^{10,11}$ Efforts to change antibiotic prescribing through the use of behavioral insights have been implemented recently in the USA and also in the UK. ${ }^{12,13}$ In the USA, a multi-site cluster randomized clinical trial, the BEARI study, evaluated the effectiveness of behavioral interventions on the rates of inappropriate antibiotic prescribing in primary care practices with existing electronic health records systems in Boston and Los Angeles. ${ }^{12}$ The interventions implemented were the following: (1) Suggested Alternatives, which utilizes computerized clinical decision support to suggest non-antibiotic treatment choices in lieu of antibiotics; (2) Accountable Justification, which prompts entry of free-text justification that become part of the patient's electronic health 
record when antibiotics are prescribed; and 3) Peer Comparison, which sends a periodic email to prescribers about his/her rate of inappropriate antibiotic prescribing relative to colleagues. The study found that all three interventions led to absolute reductions of 16-18\% in inappropriate antibiotic use over an 18-month period.

Previous cost-effectiveness analyses on reducing inappropriate outpatient antibiotic prescriptions among outpatients have focused on the cost impact of using biomarker point-ofcare tests (C-reactive protein (CRP), procalcitonin) to identify patients with possible bacterial lower respiratory tract infection. ${ }^{14-16}$ These models have shown that such tests do not significantly increase costs nor impact patients, while having a significant effect on reducing inappropriate antibiotic prescriptions, and are thus cost-effective. No other cost-effectiveness models have assessed other interventions to reduce outpatient antibiotic prescribing. To assess the tradeoffs between costs and benefits and inform public policy, we conducted a costutility analysis from the US societal perspective to determine the BEARI interventions' value in reducing inappropriate antibiotic prescriptions.

\section{Methods}

Model Background. Each of the interventions was compared to a control of no intervention, based on the assumption that in clinical practice, the baseline standard of care is the lack of targeted interventions to reduce inappropriate antibiotic use. Because the BEARI interventions focused on reducing inappropriate antibiotic use for nonspecific upper respiratory tract infections commonly caused by viruses, we included the following primary ARIs that could result in justifiable antibiotic use in adults: acute otitis media, sinusitis, and pharyngitis. ${ }^{17}$ Statistics and model parameters for these infections and resistance rates were based on data for Streptococcus pneumoniae, as this is the most common causative bacterial pathogen for community-acquired respiratory tract infections. ${ }^{18}$ We assumed that an individual could be infected with either susceptible bacterial strains, i.e., likely to clinically resolve with just one course of antibiotics, or resistant ones that may require multiple courses of antibiotic treatment.

Model Structure. We constructed a Markov model with annual cycles to simulate utilization of antibiotics, cost of care, and health outcomes for a 45 -year-old adult presenting to a healthcare provider with signs and symptoms of ARI potentially with complications, as this was the approximate average age of the population in the BEARI trial, and for which age-specific data on inappropriate antibiotic prescribing rates were available (Fig. 1). The model framework was identical for each treatment arm (control, Suggested Alternatives, Accountable Justification, and Peer Comparison), with treatment-specific model inputs. We used this model to estimate the cumulative costs, quality-adjusted life years (QALYs), and incremental cost-effectiveness ratios (ICERs) of three interventions relative to the control of no intervention over a 30-year period and from the US societal perspective. This time horizon was used as the estimated duration for amortization of any costs associated with the initial implementation of the interventions. Model computation was done in $\mathrm{R}$ version 3.3.1 using the markovchain package. ${ }^{19}$

The model was split into two major groups: those vaccinated against pneumococcal disease and those who are not. An individual began as unvaccinated and transitioned to the vaccinated group at age 65 and older at a rate based on the overall change in pneumococcal vaccination coverage per year. Within each group, the individual could contract either a viral ARI, susceptible bacterial ARI (sinusitis, otitis media, pharyngitis), or resistant bacterial ARI, due to the most common pathogens associated with these diseases in adults including $S$. pyogenes (pharyngitis), S. pneumoniae, $H$. influenzae, and M. catarrhalis (otitis media, sinusitis) and S. aureus in some cases of sinusitis. ${ }^{20,21}$ In all three clinical conditions, individuals who received antibiotics were at risk for experiencing drug-associated adverse reactions that either self-resolved or resulted in an emergency department visit and very rarely, death. Severe bacterial ARI was assumed to be one requiring hospitalization regardless of organism susceptibility, with complications such as mastoiditis and brain abscess (acute otitis media), orbital infection (sinusitis), or rheumatic heart disease, tonsillar/retropharyngeal abscess, and glomerulonephritis (pharyngitis). ${ }^{22,23}$

Probabilities. Annual transition probabilities for each state were derived from available literature and information regarding resistance patterns from CDC (Table 1). The baseline probabilities of receiving an antibiotic for undifferentiated viral infection, sinusitis, acute otitis media, and pharyngitis were derived from a national study evaluating antibiotic prescriptions dispensed in the ambulatory setting, as well as the true prevalence of bacterial URI. ${ }^{24}$ Reductions in antibiotic prescribing were as reported in the BEARI study. ${ }^{12}$ Rates of hospitalization for ARI and complications related to these hospitalizations were based on the Agency for Healthcare Research and Quality (AHRQ) National Inpatient Stay (NIS) data on ED to hospital admissions for ARIs, as well as expert opinion from infectious diseases clinicians. ${ }^{27,38}$ Rates of respiratory antibiotic-associated adverse drug reactions (ADRs) and anaphylaxis were derived from several national studies evaluating emergency department visits for antibiotic-associated ADRs; these included skin reactions, digestive effects, and central nervous system effects, among others, ranging from 12 to $17 \%$ each for macrolides, fluoroquinolones, and beta-lactams. ${ }^{1,2,26}$ Baseline rates of antibiotic resistance, as well as rate of susceptible to resistant strain conversion, were based on data from the CDC's Active Bacterial Core Surveillance Report. ${ }^{25}$ In addition, the rate of pneumococcal vaccination was based on CDC Behavioral Risk Factor Surveillance System (BRFSS) Reports. ${ }^{31}$ Finally, baseline age-adjusted mortality based on actuarial life tables 


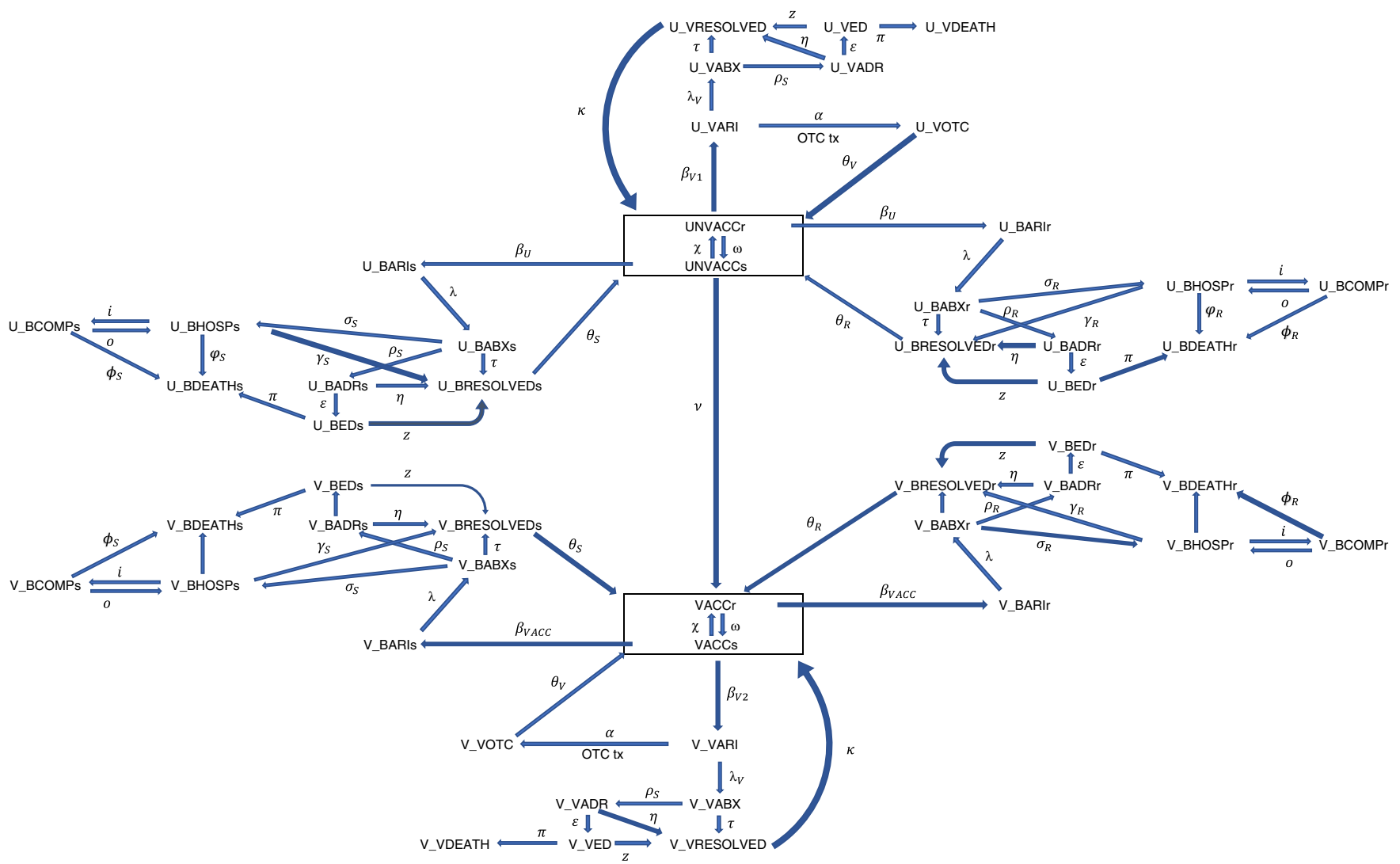

Figure 1 Markov Model Structure. Figure 1 depicts the Markov framework. "U " and "V " designate unvaccinated (UNVACC) and vaccinated (VACC) individuals, respectively, while "s" and " $r$ " subscripts represent carriers of susceptible or resistant bacterial strains. As individuals get vaccinated over time, they move from the UNVACC to the VACC pool. An individual may contract one of three types of infections: viral acute respiratory infection (VARI), susceptible bacterial acute respiratory infection (BARIs), or resistant bacterial acute respiratory infection (BARIr). For VARI, treatment is either over-the-counter and symptomatic treatment (VOTC), or inappropriate antibiotics (VABX), which may lead to an adverse drug reaction (VADR) and possible emergency department visit (VED) and/or anaphylactic death (VDEATH). Otherwise, the infection will resolve (VRESOLVED) and patients return to the pool of unvaccinated/vaccinated individuals. For BARI, all individuals should receive antibiotics (BABX), which may also lead to subsequent adverse drug reaction (ADR) and emergency visit (BED). In addition, the infection may become severe requiring inpatient hospitalization (BHOSP) and possible infectious and hospitalization complications (BCOMP). Not shown is background mortality, which assumes that individuals may exit the model at any state due to death from natural causes.

was incorporated to account for death from other causes in addition to deaths resulting from hospitalization and/or complications of S. pneumoniae infection. ${ }^{39}$

Costs. Costs were in 2016 US dollars (USD) and derived from the literature and the Centers for Medicare and Medicaid Services (CMS) reimbursement for outpatient encounters based on Common Procedural Terminology (CPT) codes. Costs included intervention implementation costs, provider office visit for respiratory infection, average cost of over-the-counter and symptomatic treatment for acute respiratory infections, and average antibiotic costs. Hospitalization and complication costs were also included for susceptible vs. resistant infections. Costs for the BEARI interventions were calculated based on the Bureau of Labor Statistics compensation rate for physicians, and the approximate amount of time a clinician would spend on an encounter if a BEARI alert or email was generated. Cost of an outpatient encounter was based on the CMS Physician Fee Schedule Healthcare Common Procedure Coding System (HCPCS) code for a minor self-limiting problem. Average over-the-counter, symptomatic treatments and antibiotic costs for acute respiratory infections were based on literature estimates, as well as hospitalization costs for acute respiratory infections and related complications. ${ }^{2,27}$

All costs were adjusted to 2016 USD using the Medical Consumer Price Index (CPI) as shown in Table 1. An annual discount rate of $3 \%$ was applied to all costs.

Quality of Life. Quality of life (QOL) utility weights were based on literature related to acute respiratory infections. For non-infected individuals, a baseline utility value of 0.87 was used across all groups. ${ }^{33}$ Acute respiratory infections were assigned a utility of 0.684 (range $0.671-0.696) .{ }^{34,35}$ Treatment for acute respiratory infections was assigned a utility of 0.814 (range 0.8010.825 ) for over-the-counter and symptomatic treatment, 
Table 1 Model Inputs

\begin{tabular}{|c|c|c|}
\hline Key transition probabilities & Base case (range) & Reference \\
\hline \multicolumn{3}{|l|}{ Probability of inappropriate antibiotics, age $20-64^{*}$} \\
\hline Control & $0.430(0.367-0.495)$ & 24 \\
\hline Suggested Alternatives & $0.119(0.101-0.137)$ & 12 \\
\hline Accountable Justification & $0.096(0.082-0.111)$ & 12 \\
\hline Peer Comparison & $0.080(0.068-0.092$ & 12 \\
\hline \multicolumn{3}{|l|}{ Probability of inappropriate antibiotics, age $\geq 65^{*}$} \\
\hline Control & $0.394(0.272-0.531)$ & 24 \\
\hline Suggested Alternatives & $0.109(0.075-0.147)$ & 12 \\
\hline Accountable Justification & $0.088(0.061-0.119)$ & 12 \\
\hline Peer Comparison & $0.073(0.051-0.099)$ & 12 \\
\hline Prevalence of true bacterial infections, age $20-64$ & $0.045(0.029-0.051)$ & 24 \\
\hline Prevalence of true bacterial infections, age $\geq 65$ & $0.063(0.051-0.75)$ & 24 \\
\hline Baseline population resistance & $0.163(0-0.313)$ & 25 \\
\hline Conversion of susceptible $\rightarrow$ resistant strain & $0.013(0-0.143)$ & 25 \\
\hline Likelihood of antibiotic adverse drug reaction (ADR) & $0.15(0.05-0.25)$ & 1,2 \\
\hline Likelihood of ADR requiring ED visit & $0.102(0.034-0.17)$ & 1,2 \\
\hline Likelihood of death due to anaphylaxis & $0.003(0-0.0084)$ & 26 \\
\hline Likelihood of hospitalization for URI & $0.004(0.002-0.005)$ & 27 \\
\hline Likelihood of complications & $0.010(0-0.020)$ & expert opinion $^{28-30}$ \\
\hline Likelihood of pneumococcal vaccination $^{\dagger}$ & $0.033(0-0.065)$ & \\
\hline Costs & Base case cost (range, 2016 US Dollars) & Reference \\
\hline \multicolumn{3}{|l|}{ Implementation } \\
\hline Suggested Alternatives & $1.91(0-5.73)$ & Expert Opinion \\
\hline Accountable Justification & $3.82(0-9.55)$ & Bureau of Labor Statistics \\
\hline Peer Comparison & $0.95(0-3.82)$ & \\
\hline MD Visit (HCPCS 99212) & $35.06(28.84 \quad 44.45)$ & CMS Physician Fee Schedule \\
\hline Antibiotics (susceptible infection) & $8.65(0.17-46.20)$ & VA Federal Supply Schedule \\
\hline Antibiotics (resistant infection) & $11.11(4.19-53.00)$ & VA Federal Supply Schedule \\
\hline OTC/symptomatic treatment & $4.98(0-10.31)$ & VA Federal Supply Schedule \\
\hline Complications ${ }^{末}$ & $17,313(16,102-18,523)$ & 27,32 \\
\hline Emergency department visit & $4088(3553-4632)$ & 2 \\
\hline Health states & Base case utility (range) ${ }^{\mathrm{I}}$ & Reference \\
\hline Non-infected ("healthy") & $0.8700(0.8600-0.8800)$ & \\
\hline Upper respiratory infection & $0.8649(0.8645-0.8652)$ & 34,35 \\
\hline Antibiotic treatment ${ }^{\S}$ & $0.8682(0.8653-0.8704)$ & 34,36 \\
\hline OTC/symptomatic treatment & $0.8685(0.8653-0.8704)$ & 34 \\
\hline ED visit for infection & $0.8693(0.8686-0.8702)$ & 34,35 \\
\hline Hospitalization for severe infection & $0.8635(0.8616-0.8654)$ & 37 \\
\hline Inpatient complications & $0.8591(0.8544-0.8603)$ & 37 \\
\hline
\end{tabular}

*Probability of inappropriate antibiotics for BEARI interventions derived based on a reduction in antibiotic prescriptions relative to the rate of antibiotic prescribing reported in the study by FlemingDutra et al.

tThe probability of getting a vaccination from year to year. BRFSS data only report the total percentage of individuals who are vaccinated (vaccine coverage), not the percentage of new vaccinations each year

tIncludes mastoiditis, intracranial abscess, orbital cellulitis, peritonsillar abscess, retropharyngeal abscess, glomerulonephritis, and Clostridium difficile

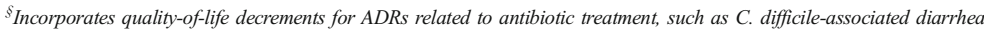

${ }^{"}$ Health state utilities are adjusted for time spent in each health state using the following equation: $Q O L_{\text {health_state }}=u_{\text {tility }}$ health_state $\times\left(\frac{\text { Duration of heath state }}{\text { One year }(365 \text { dayss) }}\right)+$ utility non-infected $\times\left(1-\frac{\text { Duration of health state }}{\text { One year }(365 \text { dayss) }}\right)$

and $0.806(0.698-0.884)$ for antibiotic prescription treatment. ${ }^{34}$ Emergency department visits were assigned a utility of 0.622 (range $0.37-0.94$ ). Hospitalization utility values were 0.53 (range $0.43-0.63$ ) for severe infections, and 0.3 (range $0.237-0.363$ ) for complications resulting from hospitalization. ${ }^{37}$ Each utility value was then used to adjust for the time spent in that health state per year to calculate an overall utility value for that health state using the following formula:

$$
\begin{aligned}
\mathrm{QOL}_{\text {health_state }}= & \text { utility }_{\text {health_state }} \times\left(\frac{\text { Duration of health state }}{\text { One year }(365 \text { days })}\right) \\
& + \text { utility }_{\text {non-infected }} \times\left(1-\frac{\text { Duration of health state }}{\text { One year }(365 \text { days })}\right)
\end{aligned}
$$

This calculated utility is shown for each health state in Table 1. Quality-adjusted life years were discounted by an annual rate of $3 \%$.
Analyses. Outcome measures for this analysis included QALYs and total costs. Treatment arms were compared to the control group in terms of cost per QALY using ICERs. The ICER is the ratio of the difference in costs to the difference in effectiveness between two alternatives ${ }^{40}$ :

$I C E R=\frac{\text { Costs }_{\text {Intervention }}-\text { Costs }_{\text {Control }}}{Q A L Y_{S_{\text {Intervention }}}-\text { Costs }_{\text {Control }}}$

The ICER allows different interventions to be compared across a standard metric. In this analysis, all treatment arms were compared to the control group. An annual discount rate of 3\% was applied to all costs and QALYs.

One-way sensitivity analyses were conducted to test the effect of individual parameters on the results of the model. Probabilities, costs, and utility values were varied per reported ranges published in the literature. Incremental costeffectiveness ratios were recalculated accordingly. 
A net monetary benefit (NMB) analysis was conducted to assess the cost-effectiveness of each therapy at varying willingness-to-pay (WTP) thresholds. ${ }^{41} \mathrm{NMB}$ is calculated as follows:

$N M B=(Q A L Y s \times W T P)-C o s t$

The NMB is determined at each willingness-to-pay threshold. The treatment with the highest NMB at a given WTP is considered the most cost-effective at that WTP threshold. An intervention is considered "dominated" if its NMB is always lower than another intervention.

Finally, we calculated the total number of antibiotic prescriptions, emergency department visits, hospitalizations, and deaths under each intervention per 100,000 uninfected individuals. We also estimated the budgetary impact of adopting these interventions by calculating the total cost to the healthcare system, should the interventions be implemented, per 100,000 individuals. The total budgetary impact for each intervention was then compared to the control group.

\section{Results}

In the base case scenario, all three BEARI interventions yielded more QALYs at a lower cost compared to the control. The QALYs yielded were 14.68 for the control group, compared to 14.73, 14.74, and 14.74 QALYs for Suggested Alternatives, Accountable Justification, and Peer Comparison, respectively, while costs were $\$ 178.21$ for the control group compared to $\$ 173.22, \$ 172.82$, and $\$ 172.52$ for each intervention, respectively. The distribution of each intervention is shown in the cost-effectiveness plane in Figure 2.

One-way sensitivity analyses revealed that the results remained robust to changes in model parameters; the BEARI interventions continued to yield more QALYs at a lower cost compared to the control group despite parameter changes, and sensitivity analysis results are thus shown as net monetary benefits (Fig. 3). Results were most sensitive to the utility of the uninfected health state, as most of the time in the model is spent uninfected. Notably, results were sensitive to the probability of experiencing an adverse drug reaction to antibiotics, and going to the emergency department for antibiotic-induced adverse drug reactions, highlighting the significant impact the interventions have on reducing exposure to adverse drug effects. Nonetheless, the BEARI interventions remained dominant over the control group. In addition, results were not affected by changes in bacterial resistance patterns.

Net monetary benefit analysis showed that the BEARI interventions all yielded marginally higher net monetary benefits compared to the control group at very low willingness-topay thresholds (Fig. 4). This effect remained consistent even at higher willingness-to-pay thresholds (not displayed on the graph for clarity).

In a population of 100,000 healthy individuals over 30 years, we expect to see a total of 160,744 antibiotic prescriptions, 265 emergency department (ED) visits, 71 hospitalizations, and 0.84 deaths due to acute respiratory infection without any intervention in place. In comparison, we expect

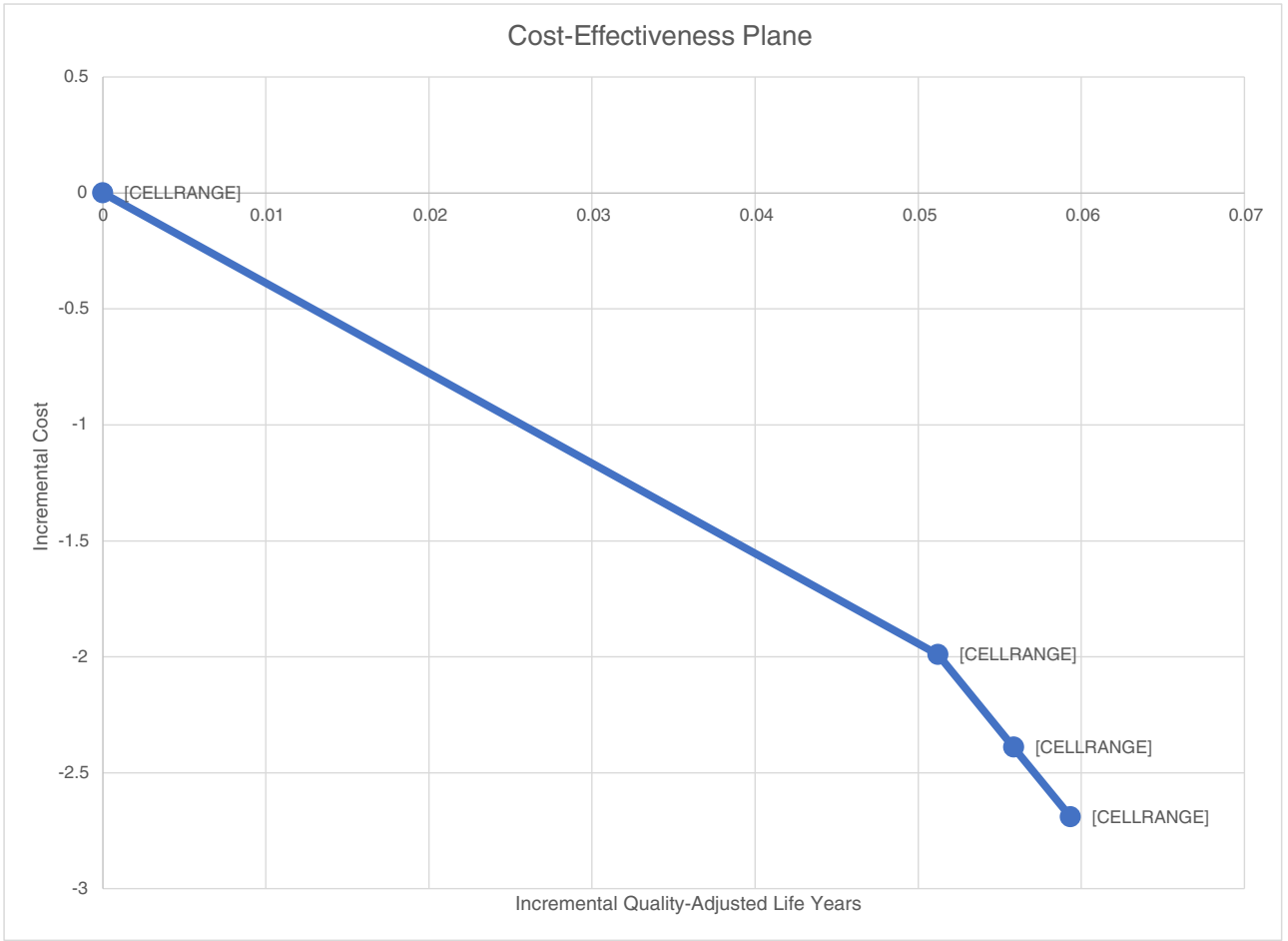

Figure 2 Cost-Effectiveness Plane. The cost-effectiveness plane depicts the incremental costs and quality-adjusted life years of each intervention relative to the control group. The further down the $X$ and $Y$-axes the intervention is, the more cost-effective it is relative to the control. 


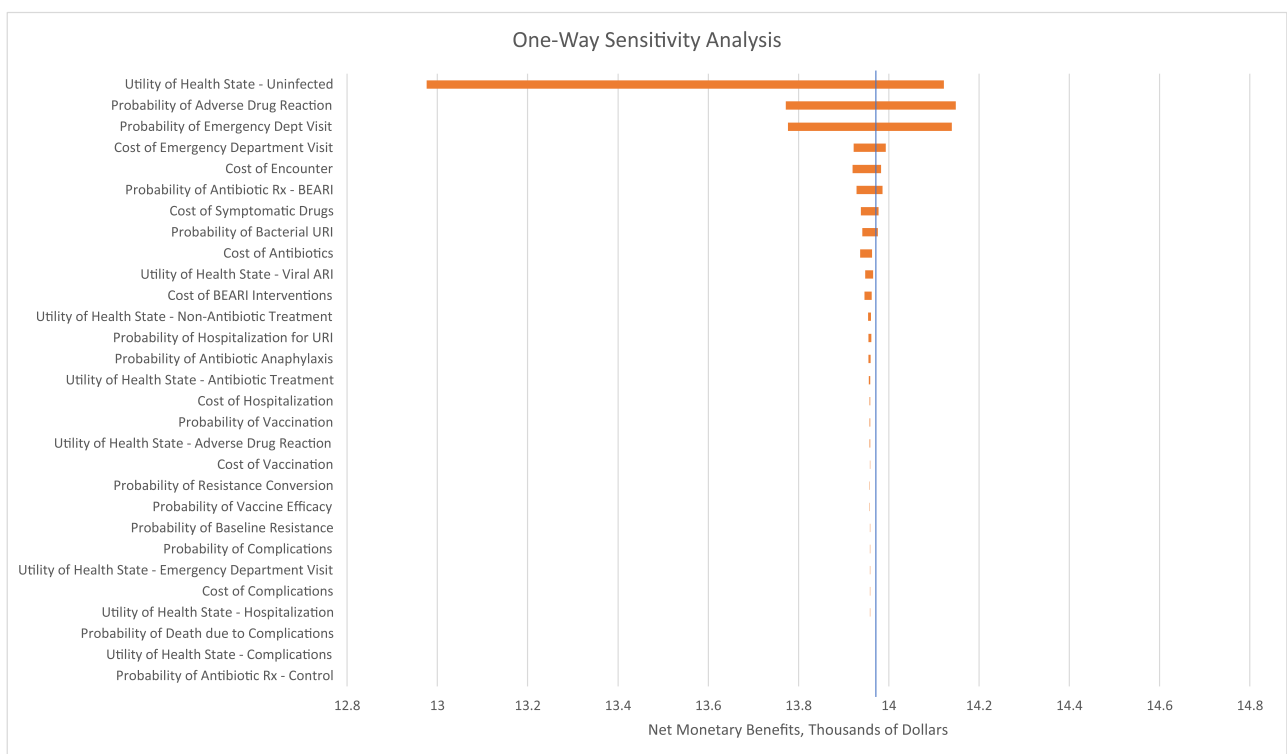

Figure 3 One-Way Sensitivity Analysis, Suggested Alternatives. One-way sensitivity analyses yielded similar trends for each intervention, with results most sensitive to the utility of the uninfected health state, followed by the likelihood and costs associated with adverse events due to antibiotics. Therefore, we have not shown a tornado diagram for each intervention evaluated. Results have been transformed into net monetary benefits as even in one-way sensitivity analyses, the interventions remained dominant over the control group, therefore yielding negative ICERs. Ranges for each parameter varied in sensitivity analysis are shown in Table 1.

just 63,830 antibiotic prescriptions, 105 ED visits, 76 hospitalizations, and 0.33 deaths under Suggested Alternatives; 56,345 antibiotic prescriptions, $92 \mathrm{ED}$ visits, 76 hospitalizations, and 0.29 deaths under Accountable Justification; and 50,828 antibiotic prescriptions, $83 \mathrm{ED}$ visits, 76 hospitalizations, and 0.27 deaths under Peer Comparison. This represents an overall budget impact of \$17.82 million for control, \$17.32 million for Suggested Alternatives, $\$ 17.28$ million for Accountable Justification, and \$17.25 million for Peer Comparison.

\section{DISCUSSION}

All the BEARI interventions are cost-effective, yielding lower costs for more QALYs compared to no intervention. There were also less antibiotic prescriptions, ED visits, and deaths under the BEARI interventions. Model results were most sensitive to the likelihood of ED visits and antibiotic-associated adverse events, yet each intervention remained cost-effective even when these probabilities were varied per reported ranges. This reduction in costs highlights the significant impact that reducing ED visits

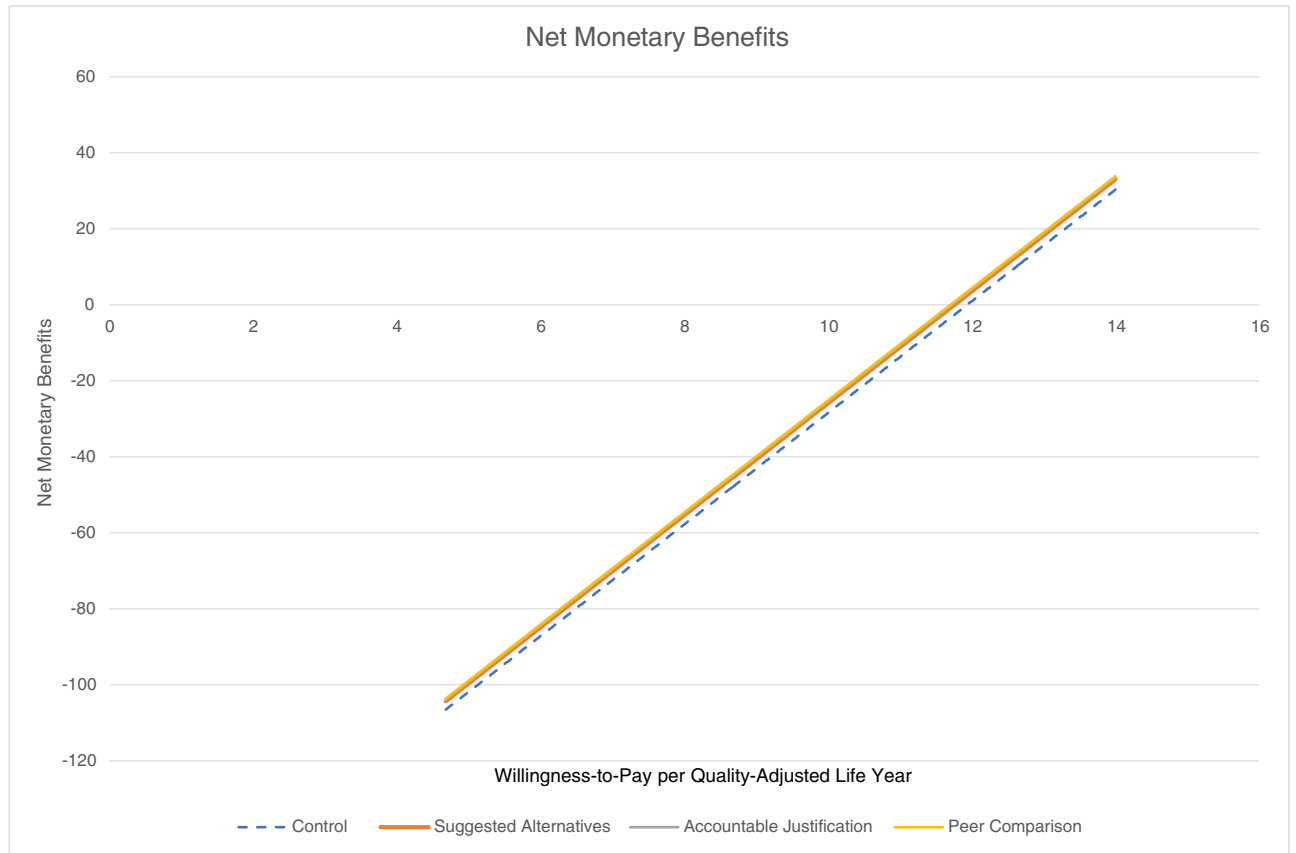

Figure 4 Net Monetary Benefits. Net monetary benefits indicate that all BEARI interventions provide greater benefit than the control group. Note that the lines depicting each intervention are essentially overlapping. The axis and graph have been scaled for graphical clarity. 
and antibiotic-associated adverse events can have on reducing overall healthcare costs. This suggests that reducing inappropriate antibiotic prescriptions can substantially affect healthcare resource utilization beyond simply improving clinical practice. Overall, patient care would benefit from any intervention that minimizes the likelihood of substandard clinical practice, patientlevel adverse drug events, and hospital and individual costs to treat them. Furthermore, the cost effectiveness of these BEARI interventions is consistent with evidence showing that behavioral economics can and should be used to design effective policies and programs to improve health, education, and the economy. ${ }^{42}$

Our data extend the existing few data available on the costeffectiveness of active antimicrobial stewardship in the adult primary care healthcare setting for URI. Previous studies utilized models that did not capture the impact of changes in antibiotic resistance resulting from the effects of an antibiotic stewardship intervention, ${ }^{14-16}$ except for one model, which attempted to quantify the cost of resistance associated with each antibiotic prescription dispensed. However, these calculations were rough estimates that could not be verified for accuracy and thus were not considered for use in our analysis. Our model is also one of few cost-effectiveness analyses in health information technology that includes a full accounting of costs and outcomes of the intervention implemented; many previous studies evaluating health information technology have only provided cost data without a full economic evaluation that includes outcomes, and particularly, standardized outcomes (such as quality-adjusted life years). ${ }^{43,44}$ In contrast, our model provides a full economic evaluation of the technology used to implement the interventions.

There are some limitations to our analysis. We were forced to make assumptions for which few or only non-robust data are available. Nonetheless, the variables identified as the major cost drivers were based on credible national datasets (ED visits and ADR information). We also did not include children in this analysis, as the referent BEARI trial included only those > 18 years of age. Children, however, represent a large proportion of inappropriate antibiotic utilization in the USA. Their inclusion in our analysis may have revealed an even larger economic impact of the BEARI intervention. Although we did not find that changes in drug resistance were an important driver of costs, our model was unable to include the potential effects of antibiotic use in animals, which has been shown to contribute to resistance patterns in humans. ${ }^{45}$ Another limitation is the assumption that resistance rates stay constant over time. Per CDC's Active Bacterial Core (ABC) Surveillance Report, rates of bacterial resistance have fluctuated widely over the past 10 years, but with a net increase of $1.2 \%$ resistant isolates per year. One-way sensitivity analyses allowed testing of resistance rates based on historical trends, and model results remained robust. It is notable that improving prescribing behavior is highly successful in the control of antimicrobial resistance in the hospital setting, but available data suggest that the impact on reversing resistance in the community setting is unlikely or at best, likely to occur at a very slow rate. $^{46,47}$ Only two studies have evaluated this issue and neither showed an impact on pneumococcal antimicrobial resistance in the community. ${ }^{48,49}$ Regardless, appropriate antibiotic prescribing can reduce healthcare costs by up to $20-30 \%{ }^{9}$

A practical concern is that the BEARI interventions will not have persistent effects on decreasing antibiotic prescribing rates or that they will be less effective in a non-experimental setting. In our analysis, the rates of antibiotic prescribing were varied based on confidence intervals reported in the randomized controlled trial to verify if improved rates of antibiotic prescribing among the control group would affect the costeffectiveness of the other interventions. Even so, the interventions continue to remain cost-effective primarily because of the significant impact that even a small reduction in antibiotic prescribing would have on adverse drug events and associated emergency department visits.

A major benefit of the BEARI interventions is the ease of implementation and the lack of a need for point-of-care blood testing (e.g., procalcitonin, CRP) in patients who usually have an uncomplicated ARI at presentation. While an underlying assumption for this model is that an existing electronic health record is already in place, thus making the implementation of the interventions inexpensive with little overhead cost, $87 \%$ of all office-based physicians and $96 \%$ of non-federal hospitals in the USA had an electronic health record system in $2015 .{ }^{50}$ The high adoption rate of electronic health record systems allows any electronic interventions to be implemented with due diligence and efficiency without the need to install a completely new system simultaneously. The few remaining organizations lacking electronic health records are likely to eventually convert as the benefits may outweigh the upfront costs. ${ }^{51,52}$

\section{Conclusion}

In this cost-effectiveness analysis, the BEARI interventions were all shown to be cost-effective relative to the control group, assuming an existing electronic health record is in place. We believe our data are robust and reveal the costeffectiveness of each BEARI intervention; a complement to prior work noting its potential to facilitate improved patient care for those with ARIs, minimize adverse outcomes associated with inappropriate antibiotic use, and potentially mitigate against the development of drug resistance.

Acknowledgements: $C L G, K M Z$, and JWH made substantial contributions to the conception and design of this project. CLG, KMZ, JWH, $D M$, and JND assisted with data acquisition, analysis, and interpretation of data for this manuscript. CLG drafted the manuscript, and $K M Z$, JWH, DM, and JND revised it critically for important intellectual content. All authors approved of the final version to be published. CLG is the guarantor.

The original BEARI Trial (NCTO1454947) was supported by the American Recovery \& Reinvestment Act of 2009 (RC4 AG039115) from the National Institutes of Health/National Institute on Aging and Agency for Healthcare Research and Quality (Principal Investigator: Dr. Doctor, University of Southern California). The project also benefited from technology funded by the Agency for Healthcare Research and Quality through the American Recovery \& Reinvestment Act of 2009 (R01 HS19913-01) (Dr. Ohno-Machado, University of 
California, San Diego). Data for the project were collected by the University of Southern California's Medical Information Network for Experimental Research (Med-INFER) which participates in the Patient Scalable National Network for Effectiveness Research (pSCANNER) supported by the Patient-Centered Outcomes Research Institute (PCORI), Contract CDRN-1306-04819 (Dr. Ohno-Machado). The authors are independent from these funding organizations, which played no role in design and conduct of the study; collection, management, analysis, and interpretation of the data; preparation, review, or approval of the manuscript; or the decision to submit the manuscript for publication.

Corresponding Author: Cynthia L. Gong, Pharm.D.; University of Southern California Leonard D. Schaeffer Center for Health Policy \& Economics, Los Angeles, CA, USA (e-mail: gongc@usc.edu).

\section{Compliance with Ethical Standards:}

Conflict of Interest: The authors declare no conflicts of interest.

\section{REFERENCES}

1. Shehab N, Patel PR, Srinivasan A, Budnitz DS. Emergency department visits for antibiotic-associated adverse events. Clin Infect Dis 2008;47(6):735-43.

2. Llop CJ, Tuttle E, Tillotson GS, LaPlante K, File TM, Jr. Antibiotic treatment patterns, costs, and resource utilization among patients with community acquired pneumonia: a US cohort study. Hosp Pract (1995). 2017;1-8.

3. Jones SC, Budnitz DS, Sorbello A, Mehta H. US-based emergency department visits for fluoroquinolone-associated hypersensitivity reactions. Pharmacoepidemiol Drug Saf 2013;22(10):1099-106.

4. Falagas ME, Bliziotis IA. Pandrug-resistant Gram-negative bacteria: the dawn of the post-antibiotic era? Int J Antimicrob Agents 2007;29(6):630-6.

5. Brown ED, Wright GD. Antibacterial drug discovery in the resistance era. Nature 2016;529(7586):336-43.

6. CDC. Antibiotic resistance threats. Atlanta, GA: US Department of Health and Human Services; 2013

7. CDC. About Antimicrobial Resistance: Four Core Actions to Fight Resistance. Centers for Disease Control and Prevention; 2015.

8. Ranji SR, Steinman MAS, Kaveh G, Sundaram V, Lewis R, Arnold S, et al. Antibiotic Prescribing Behavior Vol. 4: Closing the Quality Gap: A Critical Analysis of Quality Improvement Strategies. Technical Review 9 (Prepared by Stanford University-UCSF Evidence-based Practice Center under Contract No. 290-02-0017. Rockville, MD: Agency for Healthcare Research and Quality; 2006.

9. Drekonja D, Filice G, Greer N, Olson A, MacDonald R, Rutks I, et al. Antimicrobial Stewardship Programs in Outpatient Settings: A Systematic Review. Washington (DC); 2014.

10. Loewenstein G, Brennan T, Volpp KG. Asymmetric paternalism to improve health behaviors. JAMA 2007;298(20):2415-7.

11. Frolich A, Talavera JA, Broadhead P, Dudley RA. A behavioral model of clinician responses to incentives to improve quality. Health Policy 2007;80(1):179-93.

12. Meeker D, Linder JA, Fox CR, Friedberg MW, Persell SD, Goldstein NJ, et al. Effect of Behavioral Interventions on Inappropriate Antibiotic Prescribing Among Primary Care Practices: A Randomized Clinical Trial. JAMA 2016;315(6):562-70.

13. Hallsworth M, Chadborn T, Sallis A, Sanders M, Berry D, Greaves F, et al. Provision of social norm feedback to high prescribers of antibiotics in general practice: a pragmatic national randomised controlled trial. Lancet 2016;387(10029):1743-52.

14. Hunter R. Cost-effectiveness of point-of-care C-reactive protein tests for respiratory tract infection in primary care in England. Adv Ther 2015;32(1):69-85.

15. Michaelidis CI, Zimmerman RK, Nowalk MP, Fine MJ, Smith KJ. Cost-effectiveness of procalcitonin-guided antibiotic therapy for outpatient management of acute respiratory tract infections in adults. J Gen Intern Med 2014;29(4):579-86.

16. Oppong R, Jit M, Smith RD, Butler CC, Melbye H, Molstad S, et al. Cost-effectiveness of point-of-care $\mathrm{C}$-reactive protein testing to inform antibiotic prescribing decisions. Br J Gen Pract 2013;63(612):e465-71.

17. Zoorob R, Sidani MA, Fremont RD, Kihlberg C. Antibiotic Use in Acute Upper Respiratory Tract Infections. Am Fam Physician . 2012 86(9):817-22.
18. Jenkins SG, Brown SD, Farrell DJ. Trends in antibacterial resistance among Streptococcus pneumoniae isolated in the USA: update from PROTEKT US Years 1-4. Ann Clin Microbiol Antimicrob 2008;7:1.

19. Spedicato GA, Kang TS, Yalamanchi SB, Yadav D. markovchain. 0.6.6 ed: R; 2017.

20. Schwartz LE, Brown RB. Purulent otitis media in adults. Arch Intern Med 1992;152(11):2301-4.

21. Sokol W. Epidemiology of sinusitis in the primary care setting: results from the 1999-2000 respiratory surveillance program. Am J Med 2001;111 Suppl 9A:19S-24S.

22. Chow AW, Benninger MS, Brook I, Brozek JL, Goldstein EJ, Hicks LA, et al. IDSA clinical practice guideline for acute bacterial rhinosinusitis in children and adults. Clin Infect Dis 2012;54(8):e72-e112.

23. Shulman ST, Bisno AL, Clegg HW, Gerber MA, Kaplan EL, Lee G, et al. Clinical practice guideline for the diagnosis and management of group A streptococcal pharyngitis: 2012 update by the Infectious Diseases Society of America. Clin Infect Dis 2012;55(10):e86-102.

24. Fleming-Dutra KE, Hersh AL, Shapiro DJ, Bartoces M, Enns EA, File TM, Jr., et al. Prevalence of Inappropriate Antibiotic Prescriptions Among US Ambulatory Care Visits, 2010-2011. JAMA 2016;315(17):1864-73.

25. Active Bacterial Core Surveillance Report, Emerging Infections Program Network, Streptococcus pneumoniae, 2014. In: Prevention. CfDCa, ed. Centers for Disease Control and Prevention; 2014.

26. Ma L, Danoff TM, Borish L. Case fatality and population mortality associated with anaphylaxis in the United States. J Allergy Clin Immunol 2014;133(4):1075-83.

27. AHRQ. National Statistics on All Stays. Healthcare Cost and Utilization Project (HCUP): Agency for Healthcare Research and Quality; 2016.

28. Bird J, Biggs TC, Thomas M, Salib RJ. Adult acute rhinosinusitis. BMJ 2013;346:f2687.

29. Fokkens WJ, Hoffmans R, Thomas M. Avoid prescribing antibiotics in acute rhinosinusitis. BMJ 2014;349:g5703.

30. Little P, Stuart B, Hobbs FD, Butler CC, Hay AD, Campbell J, et al. Predictors of suppurative complications for acute sore throat in primary care: prospective clinical cohort study. BMJ 2013;347:f6867.

31. Centers for Disease Control and Prevention. BRFSS Prevalence \& Trends Data. National Center for Chronic Disease Prevention and Health Promotion; 2015.

32. Zhang S, Palazuelos-Munoz S, Balsells EM, Nair H, Chit A, Kyaw MH. Cost of hospital management of Clostridium difficile infection in United States-a meta-analysis and modelling study. BMC Infect Dis 2016;16(1):447.

33. Luo N, Johnson JA, Shaw JW, Feeny D, Coons SJ. Self-reported health status of the general adult U.S. population as assessed by the EQ-5D and Health Utilities Index. Med Care 2005;43(11):1078-86.

34. Bergus GR, Weber CA, Ernst ME, Ernst EJ. Do antibiotics affect the quality of life of patients with upper respiratory tract illnesses? It might depend on one's luck. Int J Clin Pract 2008;62(6):855-9.

35. Johnson FR, Banzhaf MR, Desvousges WH. Willingness to pay for improved respiratory and cardiovascular health: a multiple-format, stated-preference approach. Health Econ 2000;9(4):295-317.

36. Shupo F, Dorey J, Aballea S, McGarry T, Odeyemi II, Toumi M. PIN75 Health-Related Quality of Life of Clostridium Difficile Infection: A Methodological Contribution to Direct Utility Elicitation by TTO. Value Health 2012;15(7):A399.

37. Egger ME, Myers JA, Arnold FW, Pass LA, Ramirez JA, Brock GN. Cost effectiveness of adherence to IDSA/ATS guidelines in elderly patients hospitalized for Community-Aquired Pneumonia. BMC Med Inform Decis Mak 2016;16:34.

38. Fendrick AM, Monto AS, Nightengale B. The Economic Burden of NonInfluenza-Related Viral Respiratory Tract Infection in the United States. Arch Intern Med 2003; 163(4):487-94.

39. Administration SS. Period Life Table, 2013. In: Administration SS, ed. Actuarial Life Table: Social Security Administration; 2016.

40. Neumann PJ, Sanders GD, Russell LB, Siegel JE, Ganiats TG. CostEffectiveness in Health and Medicine: 2nd Edition. New York: Oxford University Press; 2016.

41. Stinnett AA, Mullahy J. Net health benefits: a new framework for the analysis of uncertainty in cost-effectiveness analysis. Med Decis Mak 1998;18(2 Suppl):S68-80.

42. Using Behavioral Science Insights to Better Serve the American People. Online: The White House Office of the Press Secretary; 2015.

43. Bassi J, Lau F. Measuring value for money: a scoping review on economic evaluation of health information systems. J Am Med Inform Assoc 2013;20(4):792-801. 
44. O'Reilly D, Tarride JE, Goeree R, Lokker C, McKibbon KA. The economics of health information technology in medication management: a systematic review of economic evaluations. J Am Med Inform Assoc 2012; 19(3):423-38.

45. Antibiotic Use in Food-Producing Animals. National Antimicrobial Resistance Monitoring System for Enteric Bacteria (NARMS).

46. Andersson DI, Hughes D. Antibiotic resistance and its cost: is it possible to reverse resistance? Nat Rev Microbiol 2010;8(4):260-71.

47. Andersson DI, Hughes D. Persistence of antibiotic resistance in bacterial populations. FEMS Microbiol Rev 2011;35(5):901-11.

48. Belongia EA, Sullivan BJ, Chyou PH, Madagame E, Reed KD, Schwartz B. A Community Intervention Trial to Promote Judicious Antibiotic Use and Reduce Penicillin-Resistant Streptococcus pneumoniae Carriage in Children. Pediatrics 2001;108(3):575-83.

49. Hennessy TW, Petersen KM, Bruden D, Parkinson AJ, Hurlburt D, Getty $\mathbf{M}$, et al. Changes in antibiotic-prescribing practices and carriage of penicillin-resistant Streptococcus pneumoniae: A controlled intervention trial in rural Alaska. Clin Infect Dis 2002;34(12):1543-50.

50. Electronic Health Record (EHR) Adoption. In: Technology TOotNCfHI, ed. Health Information Technology Data Summaries. The Office of the National Coordinator for Health Information Technology; 2015.

51. Gilmer TP, O'Connor PJ, Sperl-Hillen JM, Rush WA, Johnson PE, Amundson GH, et al. Cost-effectiveness of an electronic medical record based clinical decision support system. Health Serv Res 2012;47(6):2137-58.

52. Shekelle P, Morton SC, Keeler EB. Costs and Benefits of Health Information Technology. Evidence Report/Technology Assessment No. 132. (Prepared by the Southern California Evidence-based Practice Center under Contract No. 290-02-0003.) AHRQ Publication No. 06E006. Rockville, MD: Agency for Healthcare Research and Quality; 2006. 